\title{
Necrosis-induced apoptosis promotes regeneration in Drosophila wing imaginal discs
}

\author{
Jacob Klemm (D) , Michael J. Stinchfield, and Robin E. Harris (DD * \\ School of Life Sciences, Arizona State University, Tempe, AZ 85728, USA \\ *Corresponding author: Arizona State University, 427 E Tyler Mall LSE 229, Attn: Dr. Rob Harris, LSE 333, Tempe, AZ 85287-4501, USA. Email: robin.harris@asu.edu
}

\begin{abstract}
Regeneration is a complex process that requires a coordinated genetic response to tissue loss. Signals from dying cells are crucial to this process and are best understood in the context of regeneration following programmed cell death, like apoptosis. Conversely, regeneration following unregulated forms of death, such as necrosis, have yet to be fully explored. Here, we have developed a method to investigate regeneration following necrosis using the Drosophila wing imaginal disc. We show that necrosis stimulates regeneration at an equivalent level to that of apoptosis-mediated cell death and activates a similar response at the wound edge involving localized JNK signaling. Unexpectedly, however, necrosis also results in significant apoptosis far from the site of ablation, which we have termed necrosis-induced apoptosis (NiA). This apoptosis occurs independent of changes at the wound edge and importantly does not rely on JNK signaling. Furthermore, we find that blocking $\mathrm{NiA}$ limits proliferation and subsequently inhibits regeneration, suggesting that tissues damaged by necrosis can activate programmed cell death at a distance from the injury to promote regeneration.
\end{abstract}

Keywords: regeneration; cell death; Drosophila; apoptosis; gene expression; necrosis; imaginal disc

\section{Introduction}

Apoptosis has a fundamental role in shaping organisms during development and in maintaining tissue homeostasis (Fuchs and Steller 2011; Suzanne and Steller 2013). This vital cellular process acts to counter infection, suppress tumor formation and promote recovery from damage (Wong 2011; Fogarty and Bergmann 2017; Hacker 2018). These events occur because dying cells release signals that act on surrounding cells, resulting in different outcomes depending on the nature of the signals and the tissue context (Fogarty and Bergmann 2015; Fuchs and Steller 2015; Perez-Garijo and Steller 2015; Su 2015). Numerous aspects of regeneration can be dependent on this apoptotic signaling, including morphogenesis, inflammation, and tissue regrowth (Bergmann and Steller 2010; Perez-Garijo and Steller 2015; Vriz et al. 2014). In many organisms, factors released from dying cells stimulate the proliferation of adjacent cells as a replacement mechanism (Tseng et al. 2007; Chera et al. 2009; Li et al. 2010; Pellettieri et al. 2010; Vriz et al. 2014; Su 2015). This specialized form of compensatory proliferation is known as apoptosis-induced proliferation (AiP; Fogarty and Bergmann 2017), in which a number of mitogens, including WNTs, TGF- $\beta$, EGF, and Hedgehog, are released from dying cells (Fuchs and Steller 2015; Fogarty and Bergmann 2017). Upstream factors that lead to their production, such as JNK signaling and the activation of caspases, have also been described (Fogarty and Bergmann 2017; Pinal et al. 2019). Conversely, apoptosis can also promote the death of surrounding cells, a process known as apoptosis-induced apoptosis (AiA; Perez-Garijo et al. 2013; Fogarty and Bergmann 2015; Su 2015). This process also relies on JNK signaling and possibly represents a mechanism to ensure the proper coordination of cell death across a tissue (Perez-Garijo et al. 2013). Signals from dying cells can also result in non-autonomous protection from cell death, ensuring the preservation of neighboring cells after injury (Bilak et al. 2014). Thus, cell death can strongly influence the behavior of surrounding cells and consequently regulate the ability of a tissue to recover from damage.

Evidence that signals from apoptotic cells impact surrounding tissues first originated from studies of the Drosophila wing imaginal disc, a larval epithelial tissue that forms the adult wing (Huh et al. 2004; Perez-Garijo et al. 2004; Ryoo et al. 2004). The wing disc has been extensively characterized as a model for growth and development, and for its significant capacity to regenerate (Beira and Paro 2016; Hariharan and Serras 2017; Worley et al. 2012). Much of our understanding of imaginal disc regeneration has derived from GAL4/UAS-based genetic ablation tools, in which tissue-specific apoptosis is induced by a temperature change, permitting the study of regeneration in situ (Fox et al. 2020). However, these ablation tools rely on activating the apoptotic machinery to induce damage, limiting our ability to explore other types of cell death. One example is necrosis, the rapid and disordered death of a cell characterized by the loss of membrane integrity and release of cytoplasmic contents into the surrounding tissue (Fuchs and Steller 2015). Several factors released by cells undergoing necrosis have been identified and characterized as signaling molecules that might influence the behavior of surviving tissues (Bianchi 2007; Venereau et al. 2015; Patel 2018), although their role in wound recovery and regeneration is 
currently not well understood. In humans, necrosis can occur in all types of tissues and results from environmental insults like burns or frostbite, microbial infection (Hakkarainen et al. 2014; Bonne and Kadri 2017), and common ischemic events such as heart attacks and strokes (Yuan 2009; Konstantinidis et al. 2012; Radak et al. 2017). Various inherited and congenital conditions can also lead to necrosis, including joint disorders and sickle cell anemia (Milner et al. 1991; Masi et al. 2007). Thus, a better understanding of how a tissue responds to damage could advance therapeutic methods to improve healing and regeneration in many human disease and injury contexts.

Here, we have developed a method to induce necrotic cell death in the wing imaginal disc and used it to characterize the genetic response to necrosis compared with apoptosis. Our results show that wing discs can regenerate following necrosis but do so by inducing extensive JNK-independent apoptosis at a distance from the site of injury. Importantly, we demonstrate that this necrosis-induced apoptosis (NiA) is required for localized proliferation and efficient regeneration.

\section{Materials and methods Drosophila stocks and crosses}

Flies were cultured in conventional dextrose fly media at $25^{\circ} \mathrm{C}$ with $12 \mathrm{~h}$ light-dark cycles. The recipe for dextrose media contains $9.3 \mathrm{~g}$ agar, $32 \mathrm{~g}$ yeast, $61 \mathrm{~g}$ cornmeal, $129 \mathrm{~g}$ dextrose, and $14 \mathrm{~g}$ tego in 11 distilled water. Complete fly line details and RRIDs are available in the Reagent Table. Genotypes for each figure panel are listed in the Supplementary Methods File. The lines used as ablation stocks are as follows: hs-FLP; hs-p65; salm-DBD, DVE $\gg G A L 4\left(D^{N A}\right)$, hs-FLP; lexAop-GluR1 ${ }^{\text {LC8 }}$, hs-p65; salm-LexADBD, DVE $\gg G A L 4 / T(2 ; 3) S M 6 a-T M 6 B, T b\left(\right.$ C $\left.^{\text {GluR1 }}\right)$, hs-FLP; lexAop-GluR1 ${ }^{\text {Lc8 }}$, hs-p65/CyO; salm-LexADBD/TM6C, sb (DC GluR1 no GAL4), hs-FLP; lexAop-hep ${ }^{\text {CA, hs-p65; salm-DBD, DVE } \gg G A L 4 / T(2 ; 3) S M 6 a-T M 6 B, T b ~}$ $\left(D C^{h e p C A}\right)$, and hs-FLP; lexAop-rpr, hs-p65/CyO, salm-LexADBD, DVE $\gg$ GAL4/T(2;3)SM6a-TM6B, Tb (DC ${ }^{\text {rpr }}$; Harris et al. 2020). The stock $D R^{W N T}$-GFP was used as a marker for early regeneration (Harris et al. 2016). The following stocks were obtained from Bloomington Drosophila Stock Center: UAS-y ${ }^{\text {RNAi }}$ (BL\#64527), UAS-p35 (BL\#5073), PCNA-GFP (BL\#25749), UAS-bsk ${ }^{\text {RNAi }}$ (BL\#53310), $U_{A S-b s k^{D N}}$ (BL\#9311), UAS-egr ${ }^{\text {RNAi }}$ (BL\#58993), puc ${ }^{\text {A251 }}$ (BL\#11173), hep $^{\text {r75 }}$ (BL\#6761), UAS-hep ${ }^{\text {CA }}$ (BL\#58981), UAS-wg ${ }^{\text {RNAi }}$ (BL\#32994), UAS-Mkk4 ${ }^{\text {RNAi }}$ (BL\#35143), UAS-hep ${ }^{\text {RNAi }}$ (BL\#28710), UAS-rpr ${ }^{\text {RNAi }}$ (51846), and hid (BL\#631). CyclinE-GFP was a gift from the Wei Du lab. UAS-grnd ${ }^{\text {RNAi }}$ was a gift from the Bilder lab at UC Berkeley. UAS-miRHG and UAS-nlsGFP; egr-GAL4 were gifts from the Hariharan lab at UC Berkeley. UAS-GluR1 $1^{\text {Lc8 }}$ (Liu et al. 2014) was a gift from the Xie lab at the Stowers Institute.

\section{Generation of lexAop-hep ${ }^{\mathrm{CA}}$, lexAop-GluR1 ${ }^{\mathrm{Lc} 8}$, and DR ${ }^{\mathrm{WNT}}$-GAL80 lines}

To generate lexAop-hep ${ }^{\mathrm{CA}}$, the coding region for hep ${ }^{\mathrm{CA}}$ was cloned from UAS-hep ${ }^{\mathrm{CA}}$ stock genomic DNA and subcloned into the lexAop-GFP vector following excision of GFP, using Xhol and XbaI sites. LexAop-GluR1 ${ }^{\text {Lc8 }}$ was generated by cloning the GluR1 ${ }^{\text {Lc8 }}$ coding region from UAS-GluR1 $1^{\text {Lc8 }}$ stock genomic DNA and subcloning it into the lexAop-GFP vector following excision of the GFP, using XhoI and Xbal sites. The lexAop-hep ${ }^{\text {CA }}$ and lexAop-GluR1 ${ }^{\text {Lc8 }}$ constructs were sequence verified and inserted into the su(Hw)attP5 landing site via PhiC31 transgenesis. To generate DR ${ }^{W N T}$-GAL80, the GAL80 coding sequence was cloned from the tub-GAL80 stock genomic DNA (BL\#5132) and subcloned into the DR ${ }^{\mathrm{WNT}}$-GFP plasmid (Harris et al. 2016) following excision of the GFP using AgeI and Xbal sites. DR ${ }^{\text {WNT }}$-GAL80 was sequence verified and inserted into the attP40 and VK00027 landing sites via PhiC31 insertion. Transgenic flies were generated by embryo injections performed by BestGene, Inc.

\section{Ablation experiments}

DUAL Control experiments were performed essentially as described in (Harris et al. 2020). Experimental crosses were cultured at $25^{\circ} \mathrm{C}$ and density controlled at 50 larvae per vial. Larvae were heat shocked at day 3.5 or 4.5 of development (84 and $108 \mathrm{~h}$ after egg deposition, respectively) by placing vials in a $37^{\circ} \mathrm{C}$ water bath for $45 \mathrm{~min}$, followed by a return to $25^{\circ} \mathrm{C}$. Larvae were allowed to recover for $18 \mathrm{~h}$ before being dissected, fixed and stained, unless otherwise indicated. The non-ablating version of DUAL Control $\left(D C^{N A}\right)$ was used as a control, whereas UAS- ${ }^{R N A i}$ was used as a control for RNAi experiments. DUAL Control experiments utilized the DVE»GAL4 driver that drives expression in the majority of the wing pouch (Supplementary Figure S1, A and B). In the experiment to examine egr (Supplementary Figure S4, H-J), egr-GAL4 was utilized instead of DVE»GAL4, which drives in the adult muscle precursor (AMP) cells of the disc (Everetts et al. 2021).

\section{Regeneration scoring and wing measurements}

Wings of adult flies from heat shocked larvae were scored and measured after genotype blinding by another researcher. Scoring was performed on anesthetized adults by binning into a regeneration scoring category (Figure 1G; Harris et al. 2020). Wing measurements were performed by removing wings, mounting in Permount solution and imaging using a Zeiss Discovery.V8 microscope. Wing area was measured using the ImageJ software. Male and female adults were measured separately to account for sex differences in wing size using a reproducible measuring protocol that excludes the variable hinge region of the wing (details of measuring protocol available on request). Statistics were performed using GraphPad Prism 9.0.

\section{Immunohistochemistry}

Larvae were dissected in 1 x PBS followed by a 20-min fix in 4\% paraformaldehyde in PBS. After 3 washes in $0.1 \%$ PBST ( 1 x PBS + $0.1 \%$ Triton-X), larvae were washed in $0.3 \%$ PBST and then blocked in $0.1 \%$ PBST with $5 \%$ normal goat serum (NGS) for $30 \mathrm{~min}$. Primary staining was done overnight at $4^{\circ} \mathrm{C}$, whereas secondary staining was done for $4 \mathrm{~h}$ at room temperature. The following primary antibodies were obtained from the Developmental Studies Hybridoma Bank: mouse anti-Nubbin (1:25), mouse anti-Wg (1:100), mouse anti-Mmp1 C-terminus (1:100), mouse anti-Mmp1 catalytic domain (1:100), mouse antiLacZ (1:100), and rat anti-DE-cadherin (1:100). Rabbit anti-DCP1 (1:1000), and mouse anti-PH3 (1:400) were obtained from Cell Signaling Technologies, and chick anti-GFP (1:2000) was obtained from Abcam. The secondary anti-Chick 488 antibody was also obtained from Abcam. Other secondary antibodies, anti-rabbit 647, anti-rat 647, and anti-mouse 555 were obtained from Invitrogen. All secondary fluorophore-conjugated antibodies were used at 1:500. Images were obtained on a Zeiss AxioImager.M2 with ApoTome. For each experiment at least 15 discs were analyzed prior to choosing a representative image. Images were processed using Affinity Photo and Affinity Designer.

\section{Propidium iodide staining and TUNEL assay}

Propidium iodide (PI) staining was achieved by incubating freshly dissected wing imaginal discs in Schneider's Media with $15 \mu \mathrm{M}$ PI 

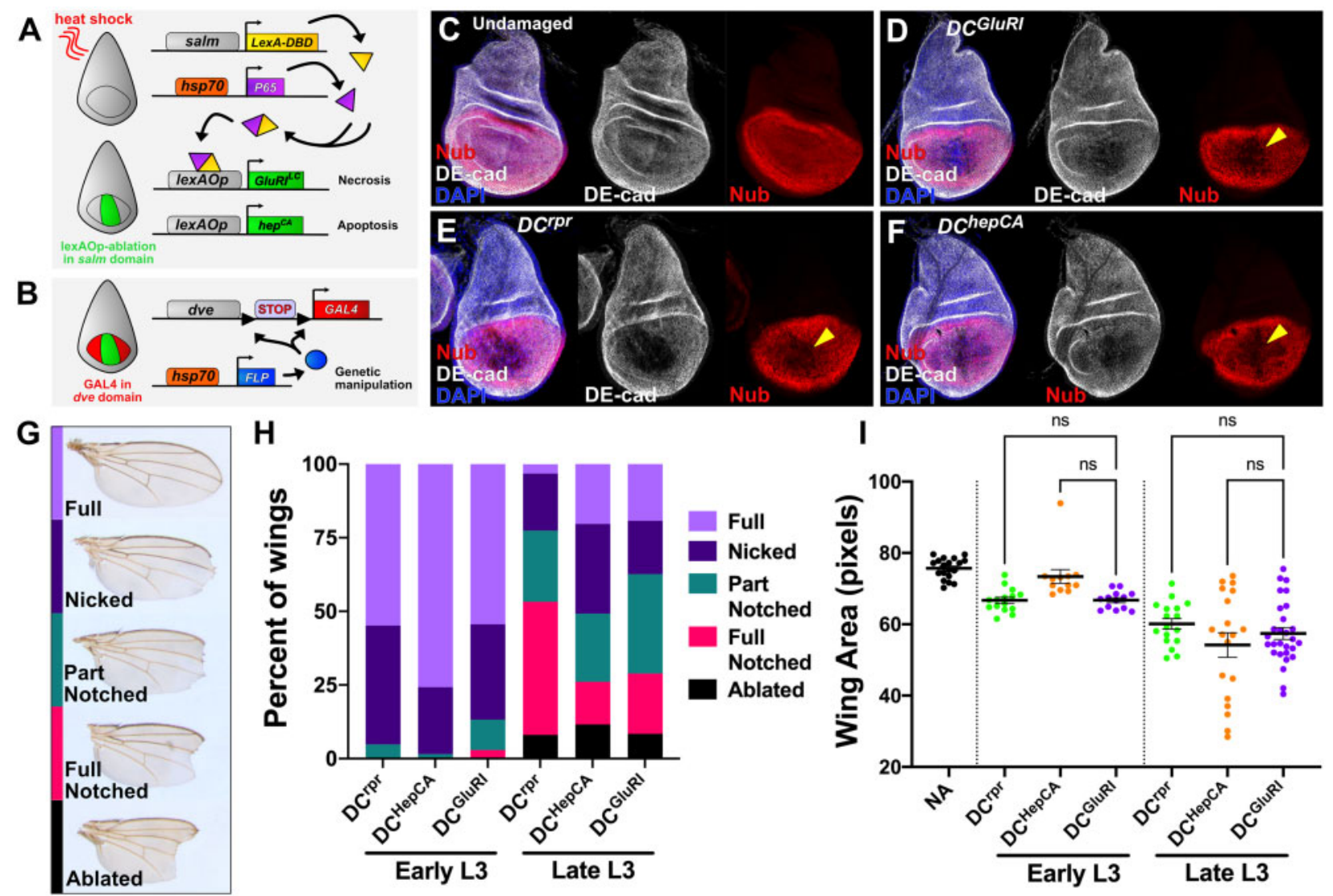

Figure 1 Drosophila wing imaginal discs regenerate comparably following ablation with $D C^{\text {rpr }}$, DC hepCA , and DC GluR1. (A, B) A schematic of the DUAL Control genetic ablation system. This system is based on a split LexA transcriptional activator, which can form a complete transcriptional activator (yellow/purple protein) using complementary leucine zipper domains following a heat shock. This in turn induces lexAop-driven ablation in the salm domain (green area of disc) (A). The DVE》GAL4 driver is used to drive UAS-based transgenes in the wing pouch independent of ablation (red area of disc), following the removal of a stop cassette by hs-FLP (B). Red lines in (A) indicate a heat shock. salm, spalt enhancer; DBD, DNA Binding Domain; due, defective proventriculus enhancer; FLP, FLP recombinase. (C-F) Wing discs were stained for the Nub pouch marker (red) and DE-cad (gray) in an unablated control $\left(D C^{N A}\right)(C)$ and following ablation with $D C^{G l u R 1}(D), D C^{r p r}(E)$, and $D C^{\text {hepCA }}(F)$. DAPI, blue. Arrowheads in (D-F) indicate loss of Nub pouch marker. (G) Adult wing ablation phenotypes following DUAL Control ablation (see also Harris et al., 2020). (H) Regeneration scoring of adult wings from early L3 discs ablated by $D C^{\text {rpr }}(\mathrm{n}=62), D C^{\text {hepCA }}(\mathrm{n}=66)$, and $D C^{\text {GluR1 }}(\mathrm{n}=68)$, and late L3 discs ablated by $D C^{\text {rpr }}(\mathrm{n}=62), D C^{\text {hepCA }}(\mathrm{n}=69)$, and $D C^{G l u R 1}(\mathrm{n}=83)$. (I) Mean pixel area of adult wings in an unablated control ( $\left.N A, n=19\right)$, and from early L3 ablated discs using DC $C^{\text {rpr }}(\mathrm{n}=22)$, $D C^{\text {hepCA }}(\mathrm{n}=12)$, and $D^{\text {GluR1 }}(\mathrm{n}=17)$ and late L3 ablated discs using $D C^{\text {rpr }}(\mathrm{n}=17), D C^{\text {hepCA }}(\mathrm{n}=19)$, and $D C^{\text {GluR1 }}(\mathrm{n}=28)$. ns, not significant. Data were analysed using one-factor ANOVA and Tukey's multiple comparisons test.

for $10 \mathrm{~min}$, followed by several washes in PBS and immediately mounting and imaging. Samples were imaged on a Zeiss AxioImager.M2 with ApoTome. The TUNEL assay was performed with the ApopTag Red In Situ Apoptosis Detection Kit (Millipore, S7165). Dissected larvae were fixed in $4 \%$ paraformaldehyde, followed by a 10-min wash in $75 \mu$ l equilibration buffer. Discs were then submerged in $55 \mu \mathrm{l}$ working strength TdT enzyme for $3 \mathrm{~h}$ at $37^{\circ} \mathrm{C}$. The reaction was stopped by adding $1 \mathrm{ml}$ stop/wash buffer and incubating for $10 \mathrm{~min}$ at room temperature, followed by three washes in PBS. Primary staining was done by incubating $65 \mu \mathrm{l}$ of the antidigoxygenin rhodamine overnight at room temperature.

\section{Quantification and Statistical Analysis}

Adult wing measurements were gathered using the ImageJ software. GraphPad Prism 9.0 was used for statistical analysis and graphical representation. Graphs depict the mean of each treatment, whereas error bars indicate the $95 \%$ CI. For quantification of DCP1 and PH3 positive cells, the cell counter plugin of ImageJ was used. For quantification of PCNA-GFP signal, mean fluorescence intensity in pixels was measured using ImageJ. To quantify PI, due to the high variability of signal the area of PI detection was calculated as a percent of the area of the lexAop-GFP reporter. All regeneration scoring (Figure 1H, Figure 3I-J, Figure S3C and S3I, Figure 6E-F), wing measurements (Figure 1I, Figure 3M, Figure 6G), and fluorescent quantification (Figure S2C, S2F, S5E, $\mathrm{S} 5 \mathrm{H}$ ) were repeated twice and the $\mathrm{n}$ for each experiment is indicated in the figure legends. Statistical significance was evaluated in Prism using one-sample t-test or one-factor ANOVA, corrected for multiple comparisons by Tukey's or false discovery rate (FDR).

\section{Results}

\section{A genetic method to induce necrotic injury in the wing imaginal disc}

To study the response to necrosis, we developed a method to induce necrotic damage in the larval wing disc by adapting a system we previously established to study regeneration called DUAL Control (Harris et al. 2020). This system utilizes a split version of the bacterial LexA/lexAop transcriptional regulator to transiently express a pro-apoptotic gene in the distal wing tissue under the control of the spalt (salm) enhancer following a short heat shock 
(Figure 1A). This apoptosis stimulates regeneration over the subsequent $24-48 \mathrm{~h}$, which can be quantitatively assessed by examining the degree to which the distal tip of the wing is present in the adult fly (Harris et al. 2020). We previously used lexAop-driven reaper (rpr) to induce cell death (Harris et al. 2020), and have since established another apoptosis-promoting transgene, lexAophep $^{\mathrm{CA}}$, a constitutive form of the JNK kinase hemipterous (AdachiYamada et al. 1999). To study necrosis, we used the GluR1 protein, a murine glutamate receptor that regulates $\mathrm{Ca}^{2+}$ ion entry into a cell (Kohda et al. 2000). A characterized allele of this ion channel (GluR1 ${ }^{\text {Lc8 }}$ ) confers constitutive activity, leading to $\mathrm{Ca}^{2+}$ influx, osmotic swelling and catastrophic death of the cell (Kohda et al. 2000; Yang et al. 2013). We generated lexAop-GluR1 ${ }^{\text {Lc8 }}$ and used it to build a version of DUAL Control that expresses GluR1 ${ }^{\text {LC8 }}$ in developing wing disc (Figure $1 \mathrm{~A}$ and Supplementary S1, A and B).

To test our ability to induce damage using this system, we heat shocked larvae bearing DUAL Control expressing GluR1 ${ }^{\text {Lc8 }}$ alongside discs ablated by rpr or hep ${ }^{\mathrm{CA}}$ (hereafter DC ${ }^{\text {GluR1 }}, \mathrm{DC}^{r p r}$, and $D C^{\text {hepCA}}$ ), using the pouch marker Nubbin (Nub) and the structural marker DE-cadherin (DE-cad) to evaluate disc integrity (Figure 1C). Ablation with DC $C^{\text {GluR1 }}$ leads to a loss of tissue in the salm domain, shown by an absence of both markers (Figure 1D), which is similar to the loss of tissue resulting from ablation by
$D C^{\text {rpr }}$ (Figure 1E) and DChepCA (Figure 1F). Ablation only occurs when all three transgenes that comprise the DUAL Control system are present (Supplementary Figure S1, C-F). We next examined the degree to which wing discs can regenerate following $D C^{\text {GluR1 }}$ ablation compared with $D C^{r p r}$ and $D C^{\text {hepCA. }}$. Regeneration was assayed by appearance of the adult wing, scored for the presence of the distal wing tip (Figure 1, G and H) and measurement of the total wing area (Figure $1 \mathrm{I})$. Ablation by $D C^{\text {rpr }}$ and $D C^{\text {hepCA }}$ in early L3 (84 h after egg deposition, AED) leads to significant regeneration, illustrated by both wing scoring and area (Figure 1, $\mathrm{H}$ and I). Ablation by $D C^{\text {GluR1 }}$ results in similar levels of ablation and regeneration (Figure 1, $\mathrm{H}$ and I). The ability of discs to regenerate is known to decline with developmental maturity from early to late L3 (Harris et al. 2016, 2020). Ablation by each method in late L3 (108 h AED) results in a similar loss of distal wing tissue (Figure 1, $\mathrm{H}$ and I). Together, these data show that $D^{\text {GluR1 }}$ can ablate the wing disc and induce regeneration at levels equivalent to that of apoptosis-based methods.

To characterize how DC ${ }^{\text {GluR1 }}$ induces damage, we examined ablated discs for different cell death markers. The loss of tissue in $D C^{\text {GluR1 }}$ coincides with strong propidium iodide (PI) staining (Figure 2A, arrowhead and Supplementary Figure S2, A and C), which labels cells with compromised membranes such as those
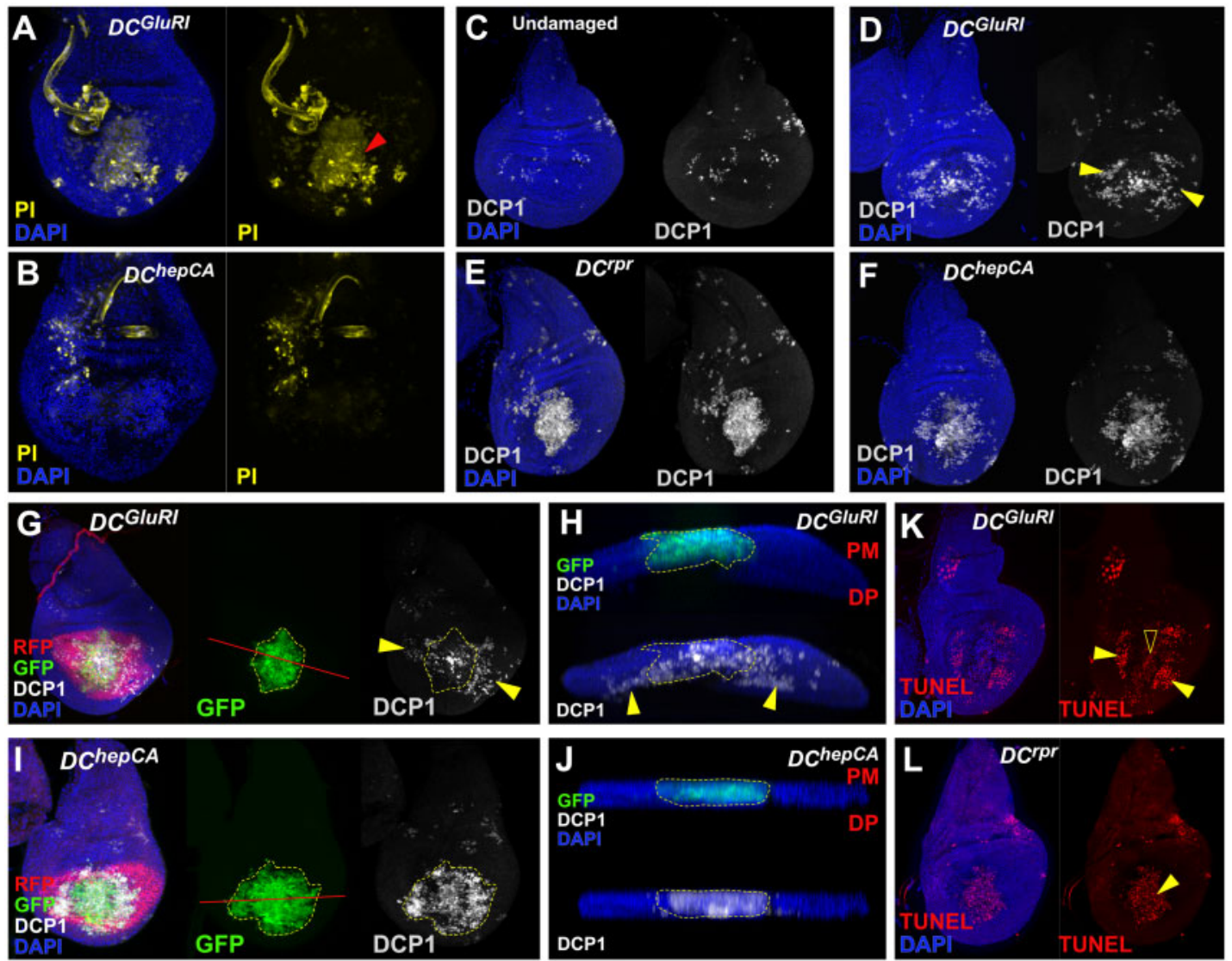

Figure 2 Ablation with $D C^{\text {GluR1 }}$ generates apoptosis at a distance. (A, B) PI staining (yellow) of DC GluR1 (A) and DChepCA (B) ablated wing discs. Arrowhead in (A) indicates PI-stained dying cells in the salm domain of the pouch. Non-specific PI staining of tracheal tubes is observed in both samples. DAPI, blue. (C-F) Caspase staining (DCP1, gray) of wing imaginal discs in an unablated control (C), and following ablation with DC $C^{G l u R 1}(D), D C^{r p r}(E)$, and DC $C^{\text {hepCA }}(F)$. DAPI, blue. Arrowheads in (D) indicate non-autonomous caspase outside of the ablation domain. (G-J) Wing imaginal discs bearing the lexAop-GFP; UASRFP reporter and ablated with DC GluR1 $(G, H)$ or DChepCA (I, J). GFP (green) labels the salm ablation domain, RFP (red) labels the surrounding unablated pouch, and DCP1 (gray) labels apoptotic cells. DAPI, blue. (G) Discs ablated with DCGluR1 show DCP1-positive cells outside of the ablation domain (arrowheads), red line indicates transverse section shown in $(\mathrm{H})$. (H) A transverse section through the pouch of the disc in $(G)$ confirms that these lateral DCP1-positive cells are not labeled with GFP. (I) Ablation by DChepCA shows almost complete overlap between the GFP and DCP1 signals, red line indicates transverse section shown in (J). (J) A transverse section of the disc in (I) confirming significant overlap of GFP and DCP1. PM, peripodial membrane; DP, disc proper. (K, L) TUNEL-labeling (red) of wing discs ablated with DC ${ }^{\text {GluR1 }}(\mathrm{K})$ and DC ${ }^{\text {rpr }}(\mathrm{L})$. Solid arrowheads indicate strong TUNEL staining in both panels, open arrowhead in $(\mathrm{K})$ indicates weak TUNEL staining present in the salm domain. 
undergoing necrosis (Wlodkowic et al. 2011). Conversely, PI staining of $D C^{\text {hep } C A}$-ablated discs shows only weak PI (Figure $2 \mathrm{~B}$ and Supplementary Figure S2, B and C). As activated caspases are a hallmark of apoptosis but not necrosis, we also stained ablated discs for the cleaved caspase DCP1. As expected, ablation with $D C^{r p r}$ or $D C^{\text {hepCA }}$ yields a strong DCP1 signal concentrated within the salm domain (Figure 2, E and F). Sporadic caspasepositive cells are seen outside of the ablation domain in all discs, likely due to the heat shock used to activate ablation (Figure 2C). Surprisingly, discs ablated with DC ${ }^{\text {GluR1 }}$ also have significant levels of DCP1 (Figure 2D). However, most of this staining is observed in the lateral wing pouch at a distance from the ablated tissue (Figure 2D, arrowheads). These DCP1-positive cells are clearly separable from the dying cells in the salm domain. They are also not a result of dying cells moving away from the site of injury, as a lexAop-GFP transgene that labels ablated cells fails to mark the caspase-positive cells outside of the salm domain (Figure 2, G and H, arrowheads and Supplementary Figure S2, D and F). This contrasts with DC hepCA discs where GFP and DCP1 almost entirely overlap (Figure 2, I and J and Supplementary Figure S2E), with very few DCP1-positive cells observed outside of the salm domain (Supplementary Figure S2F). Thus, the observed apoptosis does not occur in cells killed by GluR1 but is instead induced in neighboring cells.

In addition to promoting apoptosis, caspases can perform other cellular functions in imaginal discs unrelated to cell death (Su 2020). To examine whether the detected caspase activity is related to cell death, we also performed a TUNEL assay, which reveals strong staining in the caspase positive cells found outside of the salm domain in DC GluR1 discs (Figure 2K, arrowheads). This is consistent with GluR1-induced caspase activity leading to cell death, as in $\mathrm{DC}^{\text {rpr }}$-ablated discs (Figure 2L). We also observed a separate weaker TUNEL signal in DC ${ }^{\text {GluR1 }}$-ablated discs within the salm domain, indicative of DNA fragmentation (Figure 2K, open arrowhead), which has previously been described in necrotic cells (Grasl-Kraupp et al. 1995). Together, these results demonstrate that ablation with DC $C^{\text {GluR1 }}$ leads to tissue loss and stimulates regeneration, but also unexpectedly activates widespread apoptosis in the surrounding cells at a distance from the injury.

\section{Tissue loss via DC $C^{\text {GluR1 }}$ ablation occurs independent of caspase activity}

To understand if the non-autonomous apoptosis observed in DC $C^{\text {GluR1 }}$ discs contributes to tissue loss, or alternatively is a consequence of it, we used the baculoviral caspase inhibitor P35 to block apoptosis in the entire pouch (Hay et al. 1994). DUAL Control permits the expression of UAS-driven transgenes in the wing pouch independent of ablation under the control of a flipout GAL4 (DVE»GAL4), which is initiated by the same heat shock that triggers ablation and persists throughout the subsequent regeneration period (Figure 1B and Supplementary Figure S1, A and B; Harris et al. 2020). To test P35 in the DUAL Control system, we first blocked apoptosis in rpr-ablated discs using UAS-p35 $\left(D C^{r p r} \gg p 35\right)$. These discs have an intact pouch epithelium, shown by Nub and DE-cad staining (Figure 3A), and lack TUNEL staining (Supplementary Figure S3A). DCP1 is still detected in $D C^{r p r} \gg p 35$ discs although the intense punctate staining is replaced by a diffuse cytoplasmic signal (Figure 3, C and D), which has been reported previously when caspase activity is inhibited by P35 (Perez-Garijo et al. 2013). Conversely, P35 is unable to prevent tissue loss resulting from $D C^{\text {GluR1 }}$ ablation, indicated by the absence of Nub and DE-cad in the pouch (Figure 3B) and presence PI staining in the salm domain (Figure 3G). The appearance of the DCP1 staining in $D C^{\text {GluR1 }} \gg p 35$ discs is also diffuse (Figure 3, E and F) like that of $D C^{r p r} \gg p 35$, while TUNEL staining in the lateral pouch is now absent (Figure $3 \mathrm{H}$ ), indicating that the apoptosis in these regions is being blocked. However, the weak TUNEL signal within the cells of the salm domain is still observed (Figure $3 \mathrm{H}$, open arrowhead). These data suggest that inhibition of caspase activity cannot block tissue loss caused by DC ${ }^{\text {GluR1 }}$ ablation.

In agreement with the observed disc phenotypes, blocking apoptosis with P35 in rpr-ablated discs results in full-sized adult wings, shown by scoring and wing area measurements of both early and late L3 ablated organisms (Figure 3, I, K-K', and $\mathrm{M})$. In contrast, blocking apoptosis in $D C^{\text {GluR1 }}$-ablated discs does not prevent tissue loss, as adult wings that develop from $D C^{\text {GluR1 }} \gg p 35$ discs have similar phenotypes to discs ablated in the absence of P35 (Figure 3, J, L-L', and M). Together these observations suggest that $D C^{G l u R 1}$ can kill cells independent of caspase activity, and that the apoptotic cells seen at a distance from the wound are a consequence of this tissue loss rather than being required for it. Thus, we refer to $D C^{G l u R 1}$-induced tissue loss as necrotic ablation, and the non-autonomous caspase-positive cell death in the surrounding tissue as necrosis-induced apoptosis (NiA).

In our adult wing assays, we noted a distinct category of wings from $D C^{\text {GluR1 }} \gg p 35$ animals that are poorly patterned and have increased blistering and folding (Supplementary Figure S3, $B$ and C). This phenotype also occurs in $D C^{\text {hep } C A} \gg p 35$ discs (Supplementary Figure S3C). Blocking caspase activity with P35 in cells undergoing apoptosis can produce undead cells that continue to express mitogenic genes like wg and dpp downstream of JNK signaling, leading to tumorous overgrowth of the surrounding tissue (Perez-Garijo et al. 2004; Ryoo et al. 2004; Martin et al. 2009). Indeed, $D C^{\text {hep } C A} \gg p 35$ discs have significant ectopic $w g$ expression in the ablation domain overlapping the diffuse DCP1 compared with ablation in the absence of P35 (Supplementary Figure S3, D and E). We hypothesized that blocking apoptosis in $D C^{\text {GluR1 }} \gg p 35$-ablated discs might result in undead cells that produce the overgrown adult wing phenotype. However, in $D C^{\text {GluR1 }} \gg p 35$ discs ectopic $w g$ is only detected near to the wound edge, and not in the surrounding pouch where undead NiA would be expected to produce ectopic wg (Supplementary Figure S3F), and Dpp expression shown by a Dpp-lacZ reporter is not altered (data not shown). To test whether the observed $w g$ is important for overgrowth, we knocked it down in the entire pouch $\left(D C^{\text {GluR1 }} \gg p 35, w g^{\text {RNAi }}\right)$, which eliminated the overgrowth phenotype (Supplementary Figure S3, G-I). Since ectopic $w g$ is only associated with apoptotic cells at the wound edge it is possible that it is only these cells that contribute to overgrowth when forced to become undead. To test this, we used a DR ${ }^{W N T}$-GAL80 transgene, which takes advantage of the $D R^{W N T}$ enhancer, a damage-responsive regulatory element that drives expression in regenerating cells upon ablation (Harris et al. 2016). This transgene expresses the GAL4 inhibitor GAL80 and therefore in $D C^{\text {GluR1 }} \gg p 35, D R^{\text {WNT }}$-GAL80 discs, P35 is limited to the regions of the pouch where NiA cells occur (Supplementary Figure S3, J-L). In this experiment, the overgrowth phenotype is significantly reduced (Supplementary Figure S3C), demonstrating that NiA do not strongly contribute to overgrowth after becoming undead. Alongside the observation that they do not express $w g$, these data suggest that undead NiA cells do not activate the same genetic pathways as undead cells generated by directly activating apoptosis in the presence of P35. 

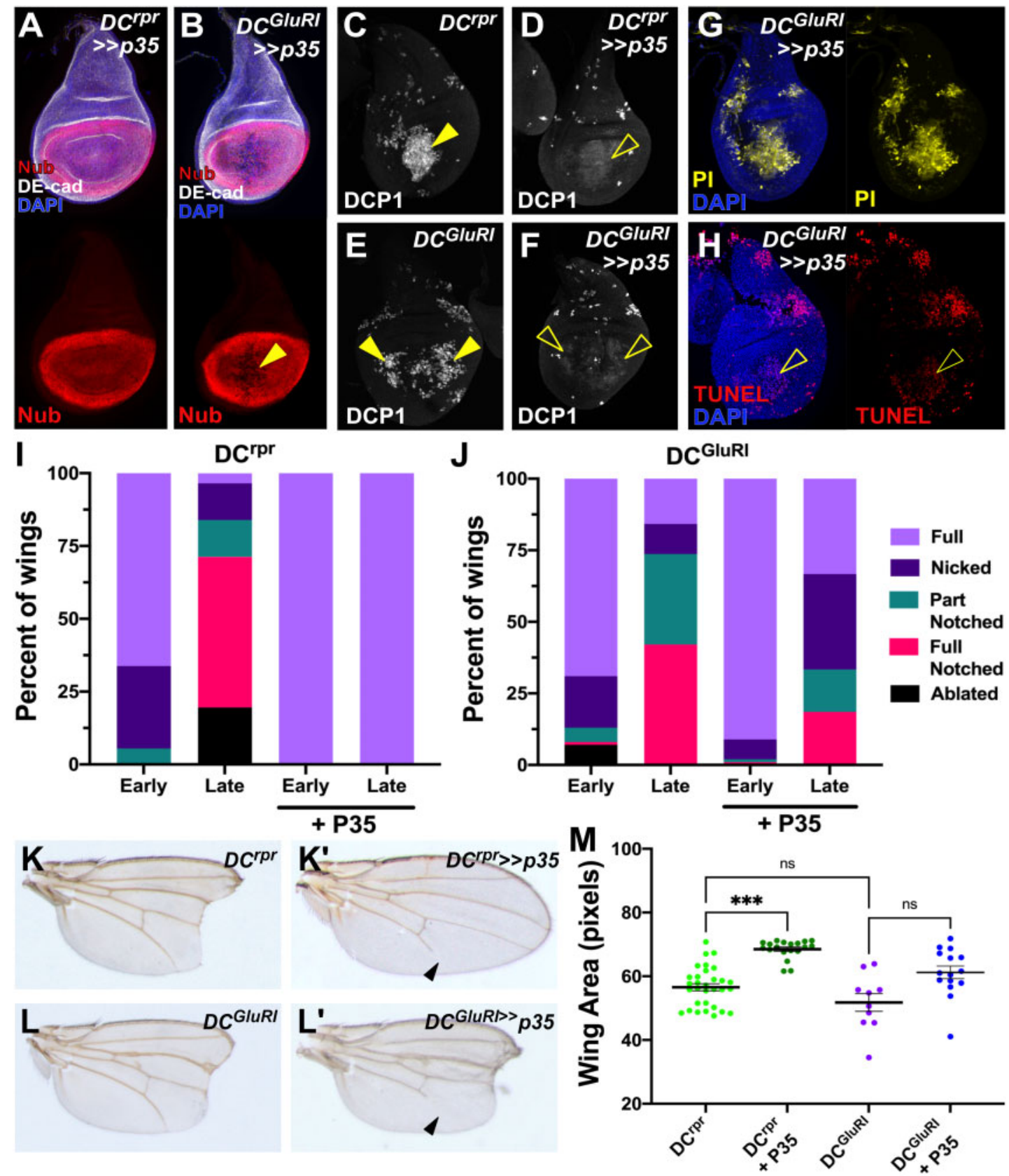

Figure 3 Inhibition of caspase activity fails to prevent ablation in DC GluR1 discs. (A, B) Wing discs stained for Nub (red) and DE-cad (gray) following ablation with $D C^{r p r} \gg p 35$ (A) and DC GluR1 $\gg p 35$ (B). DAPI, blue. Loss of tissue by DC ${ }^{\text {GluR1 }}$ ablation in the presence of P35 is indicated by the arrowhead in (B). (C-F) DCP1 staining (gray) in wing discs ablated with $D C^{r p r}(C), D C^{r p r} \gg p 35(D), D C^{G l u R 1}(E), D C^{G l u R 1} \gg p 35(F)$. The arrowheads indicate strong DCP1 staining in the ablated salm domain in $(C)$ and non-autonomous apoptosis in $(E)$, whereas the open arrowheads in $(B)$ and $(F)$ indicate the diffuse, cytoplasmic DCP1 staining in these populations that occurs in the presence of P35. (G) PI staining (yellow) of a DC GluR1 $\gg p 35$-ablated wing disc. Nonspecific PI staining of the tracheal tube is observed. DAPI, blue. (H) TUNEL-labeling (red) of a DC ${ }^{\text {GluR1 }} \gg p 35$-ablated wing disc. DAPI, blue. The open arrowhead indicates weak TUNEL staining in the salm domain, while the lateral TUNEL staining is no longer observed in the presence of P35. (I, J) Regeneration scoring of adult wings from early and late L3 discs ablated by DC ${ }^{\text {rpr }}$ (I) or DC GluR1 (J) in the presence and absence of P35. Number of wings scored: early and late L3 $D C^{r p r}(\mathrm{n}=74, \mathrm{n}=87)$, early and late L3 $D C^{r p r} \gg p 35(\mathrm{n}=110, \mathrm{n}=125)$, early and late L3 DC $C^{\text {GluR1 }}(\mathrm{n}=100, \mathrm{n}=38)$, early and late

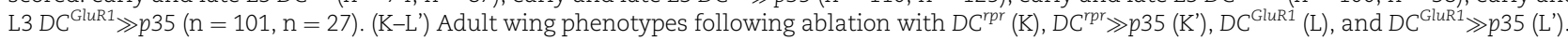
Arrowheads in ( $\left.\mathrm{K}^{\prime}\right)$ and (L') indicate an incomplete L5 vein, which is a consequence of $p 35$ expression on vein development, reported previously (PerezGarijo et al. 2004). (M) The mean area of adult wings from discs ablated with $D C^{\text {rpr }}(\mathrm{n}=31), D C^{\text {rpr }} \gg p 35(\mathrm{n}=19), D C^{\text {GluR1 }}(\mathrm{n}=10)$, and DC ${ }^{\text {GluR1 } \gg p 35}(\mathrm{n}=$ 15), ns, not significant; ${ }^{* *} P=0.0002$, data were analyzed by one-factor ANOVA and Tukey's multiple comparisons test.

\section{$\mathrm{NiA}$ and wound edge apoptosis originate separately}

To investigate the origin and timing of the NiA seen in DC GluR1 discs, we performed a time course of ablation and regeneration (Figure 4). To observe the formation of regenerating cells we took advantage of the $D R^{\mathrm{WNT}}$ enhancer driving GFP, the expression of which represents one of the earlier signaling events in disc regeneration (Figure 4, A-F; Harris et al. 2016). DR ${ }^{\mathrm{WNT}}$-GFP expression becomes apparent at the wound edge $6 \mathrm{~h}$ post-ablation with DC GluR1 (Figure 4B), while most apoptotic cells in this region become obvious at around $12 \mathrm{~h}$ (Figure 4C, arrowhead). NiA populations are still not observed at this timepoint and only occur at 

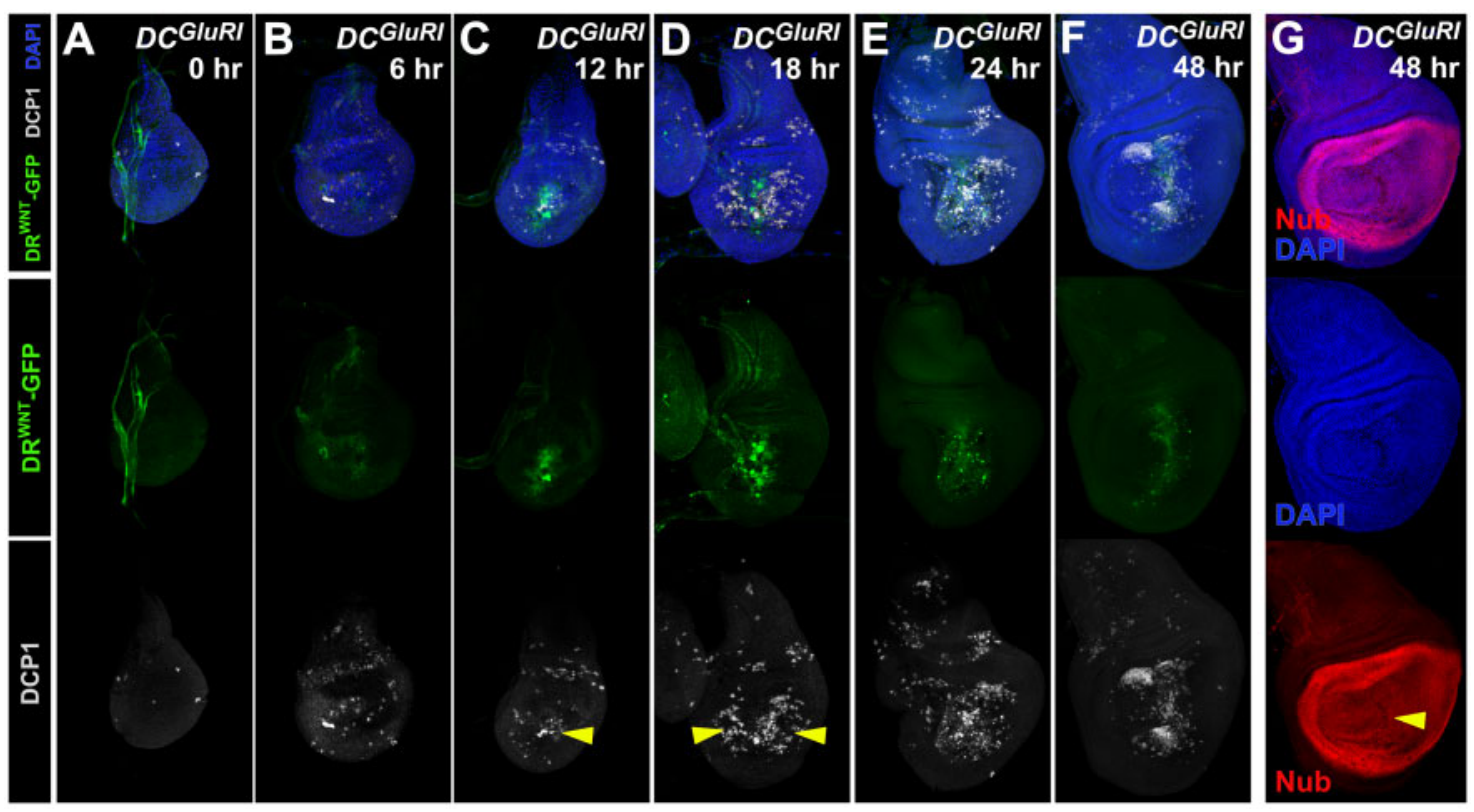

Figure $4 \mathrm{NiA}$ forms after wound edge signaling occurs. (A-F) A time course of regeneration for wing discs bearing a fluorescent reporter of a damageresponsive WNT enhancer (DR WNT -GFP, green; Harris et al. 2016), ablated with DC GluR1 and stained for DCP1 (gray) at (A) 0 h, (B) 6 h, (C) 12 h, (D) 18 h, (E) $24 \mathrm{~h}$, and (F) $48 \mathrm{~h}$ after heat shock. DAPI, blue. Arrowhead in (C) indicates wound edge apoptosis at $6 \mathrm{~h}$ coinciding with the appearance of DR ${ }^{\mathrm{WNT}}$-GFP expression, arrowheads in (D) indicate NiA at $18 \mathrm{~h}$. (G) A wing imaginal disc at $48 \mathrm{~h}$ of recovery following ablation with DC GluR1. The pouch marker Nub (red) and DAPI staining (blue) show continuity of the pouch is restored (arrowhead).

$18 \mathrm{~h}$ post-ablation after the appearance of wound edge apoptosis and $D R^{W N T}$-GFP activity (Figure 4D, arrowheads). The NiA persists as regeneration proceeds (Figure $4 \mathrm{E}$ ), and by $48 \mathrm{~h}$, when $D R^{\mathrm{WNT}}$. GFP expression is diminished, the DCP1-positive cellular debris appears to be pushed dorsally and ventrally, possibly as a result of pouch growth (Figure 4F). At this time point, the pouch is fully restored, indicated by Nub (Figure $4 G$ ). Interestingly, $D R^{W N T}$-GFP activity is never observed beyond the wound edge and does not occur in NiA cells. Thus, the time course shows that formation of NiA is not only spatially distinct from the wound edge apoptosis and damage associated signaling shown by $D R^{W N T}$-GFP, but is also temporally separated from it. These observations suggest that these two populations might arise via different mechanisms.

\section{$\mathrm{NiA}$ is independent of JNK signaling}

The $D R^{W N T}$-GFP reporter used in the time course is directly activated by JNK signaling (Harris et al. 2016). Since $D R^{W N T}$-GFP expression is restricted to the wound edge, and JNK signaling is a key pathway that regulates both apoptosis and proliferation in response to damage (Pinal et al. 2019), we evaluated JNK signaling

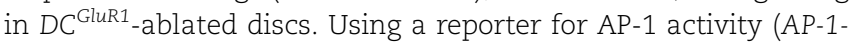
GFP; Chatterjee and Bohmann 2012), we found that JNK signaling is present only in wound edge cells and not in cells undergoing NiA (Figure $5 \mathrm{~A}$ ), consistent with the $D \mathrm{R}^{\mathrm{WNT}}$-GFP reporter expression. Examination of the JNK target genes puc, wg, and Mmp1 supports these results (Figures 5, B-D), suggesting that necrotic ablation leads to JNK upregulation and low levels of apoptosis at the wound edge, and induces NiA at a distance without activating JNK.

To test whether NiA does indeed occur independent of JNK, we investigated how altering different elements of the JNK pathway might influence either the wound edge or NiA populations in

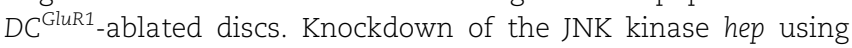
RNAi reduces the expression of the JNK target Mmp1 but does not strongly alter apoptosis at the wound edge (Figure 5E). Importantly, NiA cells are still observed in the absence of hep (Figure 5E). This result is confirmed using a hep hemizygous mutant $\left(h \mathrm{p}^{\mathrm{r} 75} / \mathrm{Y}\right)$, although the level of apoptosis across the whole disc is somewhat reduced when using this allele (Supplementary Figure S4A). The expression of a dominant negative form of the JNK basket (UAS-bsk ${ }^{D N}$ ) strongly suppresses Mmp1 expression (Figure 5F), and unlike the loss of hep, bsk ${ }^{\mathrm{DN}}$ also reduces apoptosis at the wound edge (Figure 5F), suggesting that this cell death is dependent on bsk. The population of NiA cells is also unaffected in this background (Figure 5F), supporting the idea that NiA formation does not depend on JNK signaling. RNAi knockdown of bsk also confirms these results (Supplementary Figure S4B). Since the loss of bsk, but not hep, seems to influence wound edge apoptosis, we also targeted an alternative JNK kinase, Mkk4, for knockdown in DC GluR1-ablated discs. Unlike hep, reduction of Mkk4 levels reduces both the apoptosis and Mmp1 expression at the wound edge, but again has no influence on the appearance of NiA (Figure 5G). We also reduced expression of a TNF receptor grindelwald (grnd) and TNF ligand egr using RNAi. Neither manipulation affected the apoptosis or JNK activity at the wound edge or the appearance of the cells undergoing NiA outside the ablated domain (Supplementary Figure S4, C and D). Moreover, a reporter for egr expression (egr-GAL4; UAS-GFP) shows no upregulation of egr at the wound edge or in cells undergoing NiA (Supplementary Figure S4, H-J). Interestingly, however, egr expression that is normally observed in AMP cells under the notum is reduced upon ablation (Supplementary Figure S4, H-J). Overall, these data suggest that necrotic ablation induces low levels of apoptosis and JNK signaling at the wound edge, which occurs downstream of a JNK receptor grnd but is dependent on both Mkk4 and bsk, while also generating NiA at a distance via a JNK-independent signal.

As NiA does not appear to require JNK, we sought to investigate factors upstream of the observed activated caspase DCP1 

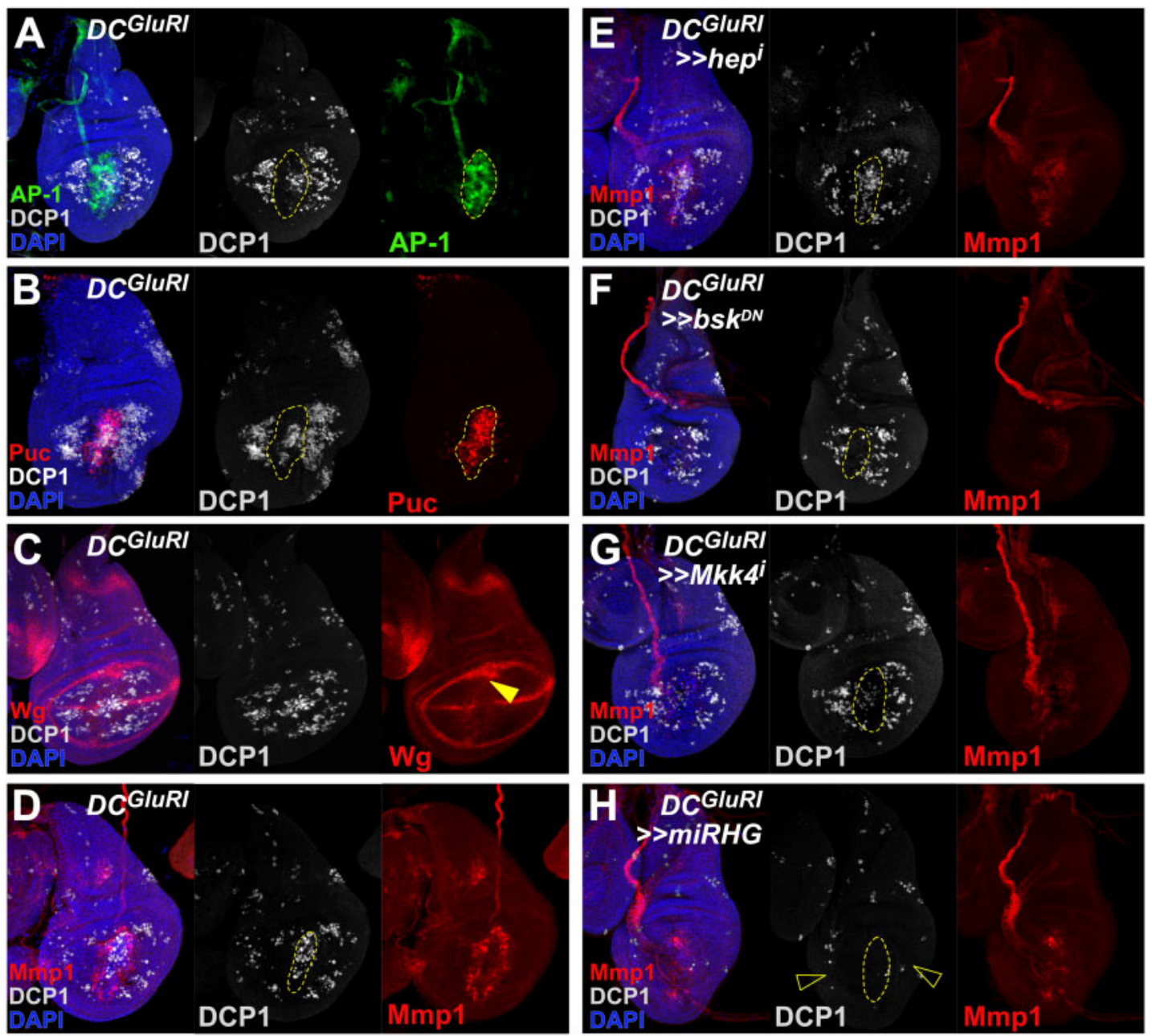

Figure $5 \mathrm{NiA}$ acts independent of the JNK pathway. (A, B) Wing discs ablated with DC GluR1 and bearing the AP-1-GFP (green) (A) and puc-lacZ (red) (B) reporters of JNK pathway activity, stained for DCP1 (gray). DAPI, blue. These reporters show strong overlap with the wound edge apoptosis, but not with the NiA cells. As in other panels, the dashed line outlines the ablated salm domain, indicated by the appearance of cells labeled by DAPI. (C, D) Wing imaginal discs ablated with DC GluR1 and stained for the JNK targets Wg (red) (C) and Mmp1 (red) (D). Arrowhead in (C) indicates ectopic Wg at the wound edge. DCP1, gray; DAPI, blue. (E) A wing imaginal disc ablated with DC ${ }^{\text {GluR1 }} \gg$ hep ${ }^{R N A i}$ stained for DCP1 (gray) and Mmp1 (red), DAPI, blue. Both the wound edge apoptosis and NiA cells remain unaffected following knockdown of hep. (F) A wing imaginal disc ablated with DC ${ }^{G l u R 1} \gg b s k^{D N}$ stained for DCP1 (gray) and Mmp1 (red). DAPI, blue. Wound edge apoptosis is largely absent, while NiA cells remain unaffected. (G) A wing imaginal disc ablated with $D C^{G l u R 1} \gg M k k 4^{R N A i}$ stained for DCP1 (gray) and Mmp1 (red). DAPI, blue. Wound edge apoptosis is strongly reduced, while NiA cells remain unaffected.

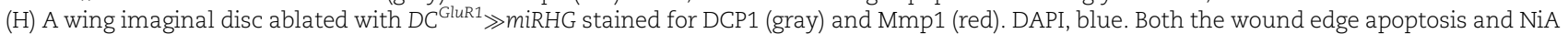
(open arrowheads) are eliminated following knockdown of rpr, hid, and grim with miRHG.

that might be required to produce NiA following necrotic ablation. We used an RNAi targeting the dIAP1 inhibitor rpr, which activates cell death immediately upstream of initiator caspases. Knockdown of rpr leads to a moderate reduction in apoptosis at the wound edge, but also reduces NiA formation (Supplementary Figure S4E). Mutation of another dIAP1 inhibitor, head involution defective (hid), did not have an effect (Supplementary Figure S4F). However, when a miRNA targeting rpr, hid and another dIAP1 inhibitor grim was used (UAS-miRHG), NiA formation was eliminated (Figure 5H), as was the cell death normally observed at the wound edge, indicating that more than one of these genes are required for both wound edge apoptosis and NiA. Importantly,

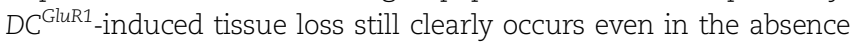
of NiA (Supplementary Figure S4G), while JNK signaling indicated by Mmp1 expression is observed, albeit at a reduced level (Figure 5H). JNK signaling is thought to activate a feedforward mechanism through Dronc, reactive oxygen species (ROS), and hemocytes (Amcheslavsky et al. 2018), and therefore this diminished Mmp1 labeling could be due to interference with this mechanism. Overall, these results suggest that necrotic ablation leads to apoptosis at the wound edge and NiA in the surrounding pouch, and although each is regulated by different activating signals, they potentially rely on the same downstream effectors, rpr, hid, and grim, to kill cells.

\section{$\mathrm{NiA}$ is required for damage-induced proliferation and subsequent regeneration}

To understand the potential contribution of NiA to regeneration, we examined how the appearance of NiA coincides with regenerative proliferation following necrotic ablation. We used reporters for E2F (PCNA-GFP) and Cyclin E (cycE-GFP), and PH3 staining to examine changes in proliferation that occur during regeneration. These markers show that proliferation induced by necrotic ablation does not significantly increase in the wing pouch until around $24 \mathrm{~h}$, after the formation of NiA at $18 \mathrm{~h}$ (Figure $6, \mathrm{~A}$ and $\mathrm{B}$ and Supplementary Figure S5, A-E). To test whether NiA regulates this localized proliferation, we sought to eliminate this apoptosis following DC ${ }^{\text {GluR1 }}$ ablation. As NiA is unaffected by blocking 

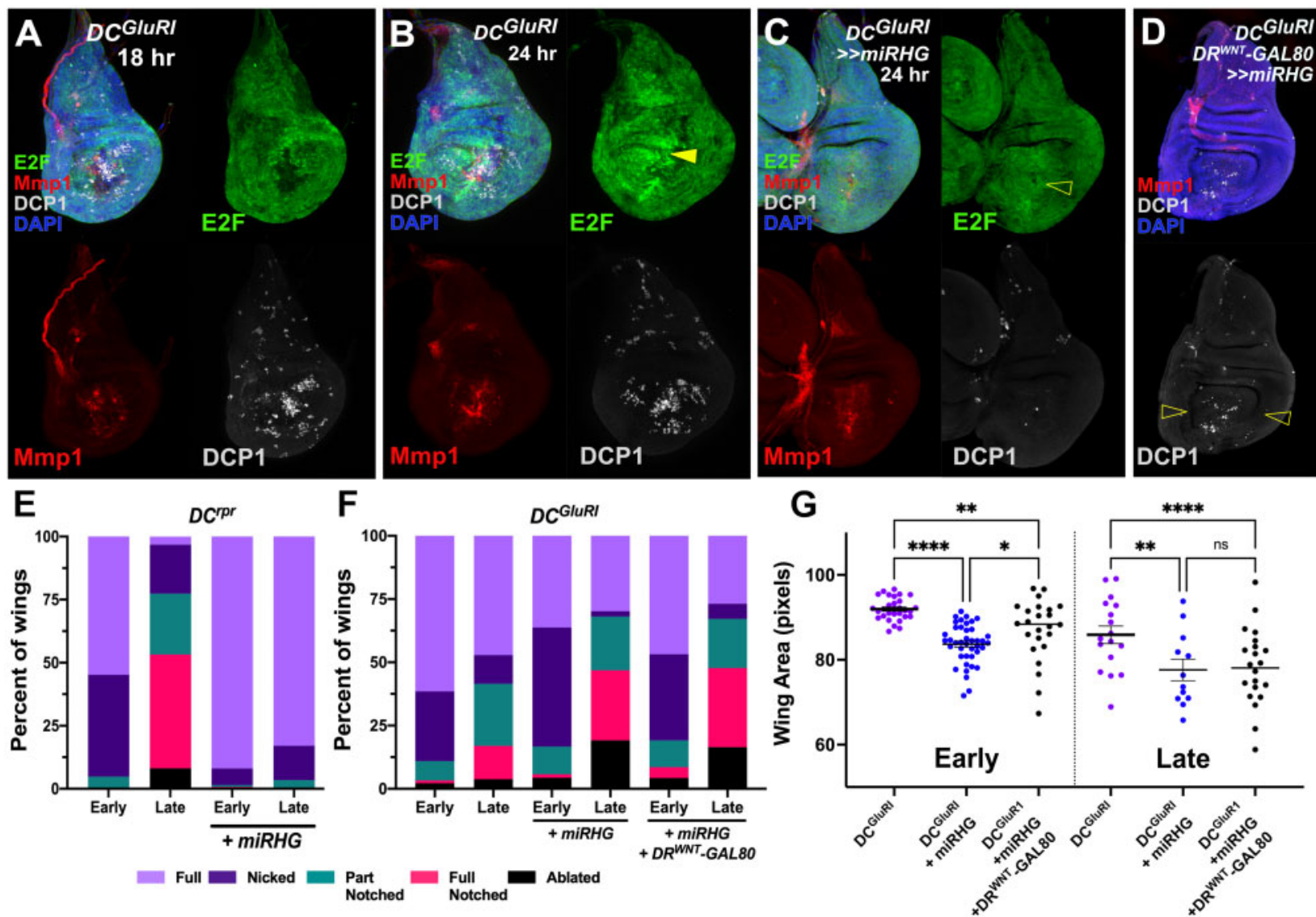

Figure 6 Blocking NiA formation impairs regeneration. (A, B) Wing discs bearing an E2F transcriptional reporter (PCNA-GFP, green) ablated with DC GluR1 and stained for Mmp1 (red) and DCP1 (gray) at $18 \mathrm{~h}$ (A) and $24 \mathrm{~h}$ (B) of recovery. DAPI, blue. Arrowhead in (B) shows the localized proliferation that is only present after $24 \mathrm{~h}$ of recovery. (C) Wing disc bearing PCNA-GFP (green) ablated with DC GluR1 $\gg$ miRHG and stained for Mmp1 (red) and DCP1 (gray), DAPI, blue. The open arrowhead indicates reduced proliferation upon miRHG expression. JNK signaling, indicated by Mmp1 staining, is still present in these discs. (D) A wing imaginal disc ablated by DC ${ }^{\text {GluR1 }} \gg$ miRHG in the presence of DR ${ }^{\text {WNT }}$-GAL80 and stained for DCP1 (gray) and Mmp1 (red). DAPI, blue. Open arrowheads indicate a loss of NiA. (E, F) Regeneration scoring of adult wings from early and late L3 discs ablated with DC ${ }^{\text {rpr }}$ (E) or DC ${ }^{\text {GluR1 }}$ (F) in the presence and absence of miRHG. Number of wings scored: early and late L3 DC $C^{r p r}(n=62, n=63)$, early and late L3 DC ${ }^{\text {pr }} \gg m i R H G(n=137, n=88)$,

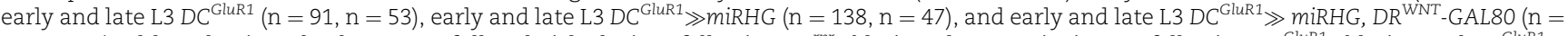
$47, n=67)$. Although miRHG leads to more full and nicked wings following $D C^{\text {rpr }}$ ablation, the opposite is seen following $D C^{\text {GluR1 }}$ ablation and $D C^{\text {GluR1 }}$

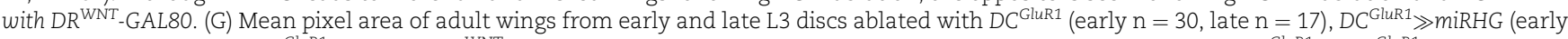

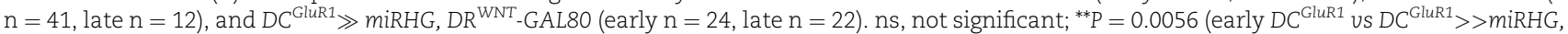
$\mathrm{DR}^{\mathrm{WNT}}$-GAL80), ${ }^{* * * *} \mathrm{P}<0.0001,{ }^{*} \mathrm{P}=0.0254,{ }^{* *} \mathrm{P}=0.0015$ (late DC GluR1 US DC ${ }^{\text {GluR1 }}>>$ miRHG), data were analyzed by one-factor ANOVA and FDR's multiple comparisons tests.

JNK signaling, and P35 allows dying cells to persist in the disc which could complicate our analysis due to the observed overgrowth (Supplementary Figure S3), we used UAS-miRHG to prevent the formation of NiA. The lack of NiA in the pouch following necrotic ablation results in a loss of PCNA-GFP upregulation that is normally seen at $24 \mathrm{~h}$ (Figure 6C and Supplementary Figure $\mathrm{S} 5 \mathrm{H}$ ), as well as diminished PH3 levels (Supplementary Figure $\mathrm{S} 5 \mathrm{E})$, suggesting that NiA is required for regenerative proliferation. Importantly, JNK signaling at the wound edge is still observed following miRHG expression, indicated by the presence of Mmp1 (Figure 6C), demonstrating that proliferation following necrotic ablation is not dependent solely on JNK activity from the wound, but also requires NiA.

We next examined how blocking NiA might influence the discs capacity to regenerate. Evaluating adult wings that develop from $D C^{\text {GluR1 }} \gg$ miRHG discs shows they have a reduced ability to regenerate compared with control discs (Figure 6, F and G). This result is significant, as blocking apoptosis with miRHG would normally be expected to improve regeneration, as it does in $D C^{r p r}$-ablated discs (Figure 6E). However, in this experiment apoptosis is blocked throughout the pouch and is not specific to the NiA. Therefore, we used DR ${ }^{\text {WNT }}$-GAL80 to limit miRHG expression solely to the surrounding pouch where NiA develops and allow wound edge apoptosis to still occur (Figure 6D). When wound edge apoptosis can still occur but NiA is blocked, regeneration is still strongly inhibited in both early and late L3 discs (Figure 6, F and $G$ ), demonstrating that NiA is specifically required for regeneration in response to necrosis.

\section{Discussion}

\section{Necrosis induces apoptosis at a distance in the wing imaginal disc}

Necrotic ablation induces apoptosis in cells in two regions of the injured disc: at the wound edge and more extensively in the surrounding pouch. These two populations of dying cells are likely induced by separate activating signals, rely on the same downstream effectors, and contribute differently to regeneration (Figure 7). The wound edge apoptosis coincides with JNK activity and is likely dependent on it, as interference with bsk strongly 


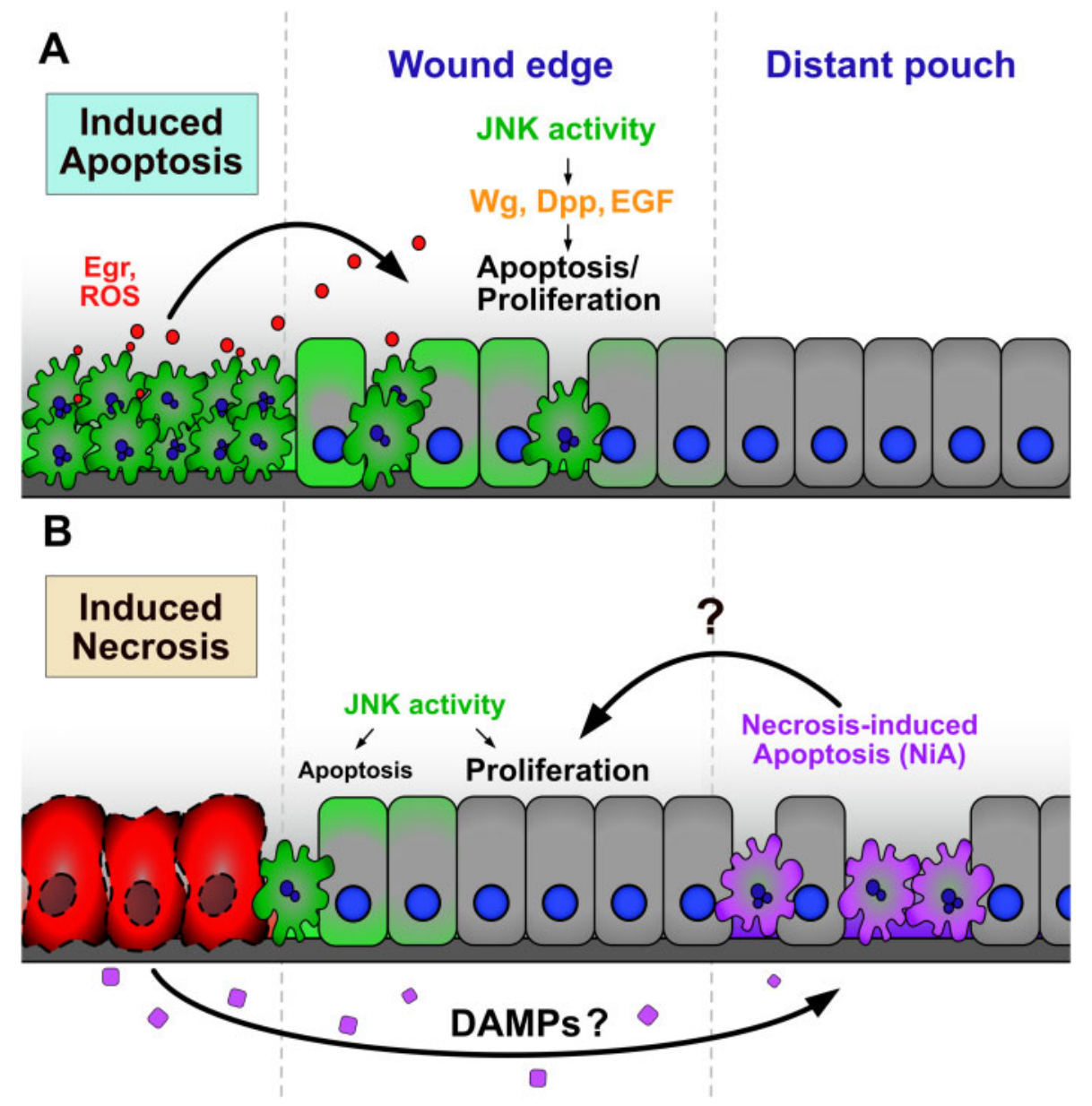

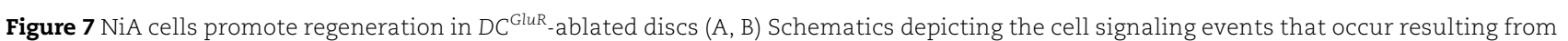
apoptotic ablation (A) and necrotic ablation (B). Previous studies have shown dying cells release ROS and promote Egr release via hemocytes and potentially other mechanisms, inducing high levels of JNK signaling at the wound edge (Fogarty and Bergmann 2017). In turn, wound edge apoptotic cells and surviving cells with lower JNK signaling release mitogens including Wg, Dpp, and EGF, to promote regenerative signaling in the adjacent wound edge tissue. In cells undergoing necrosis, low levels of JNK signaling occurs downstream of the receptor at the wound edge, likely through pathways related to polarity disruption, mechanical stress, or immediate stress signals such as ROS. This signaling induces sporadic apoptosis at the wound edge that potentially contributes to proliferation and recovery. Necrotic cells also release an as yet unidentified long-range signal that could be a DAMP, resulting in the formation of NiA cells in the distant pouch. These cells promote proliferation formation and regeneration.

limits the caspase activity observed at the wound. The factors that can activate JNK signaling, and those downstream that regulate whether cells subsequently proliferate or undergo apoptosis, are complex and context-dependent (Pinal et al. 2019; La Marca and Richardson 2020). However, our experiments show that pathway activation following necrotic ablation does not rely on a receptor for JNK, grnd, and requires the JNK kinase Mkk4 more than hep. There are a number of signals that can induce JNK activity in this way, including loss of polarity, mechanical inputs, and the release of immediate stress signals such as ROS (La Marca and Richardson 2020). ROS has been shown to upregulate both JNK and P38, another factor that regulates proliferation and apoptosis, via Ask1/AKT (Santabarbara-Ruiz et al. 2015, 2019), while ROS is known to be an important regulator of regeneration in the wing disc (Santabarbara-Ruiz et al. 2015; Brock et al. 2017; Khan et al. 2017; Santabarbara-Ruiz et al. 2019). Thus, it is possible that JNK signaling and the associated apoptosis at the wound edge induced by necrotic ablation is mediated via this pathway. It is likely that this JNK activity contributes to recovery following necrosis, as it does in apoptosis-induced regeneration. However, our observation that blocking NiA limits regeneration, despite wound edge apoptosis and JNK being unaffected, suggests that regeneration does not solely depend on JNK signaling.

The signals that lead to the NiA at a distance from the necrotic injury are less clear, however. A related phenomenon to the one we have observed, in which cells undergoing programmed cell death induce additional non-autonomous apoptosis, has been described in the wing imaginal disc and the mouse hair follicle (Perez-Garijo et al. 2013). However, in both contexts these events are induced by apoptotic cell death rather than necrosis, and are mediated by JNK signaling, unlike NiA. In a parallel observation to that of apoptotic cells communicating with surrounding tissues, studies have shown that cells undergoing necrosis might also release factors that regulate the behavior of neighboring cells. These factors are collectively known as damage-associated molecular patterns or DAMPs (Roh and Sohn 2018; Gong et al. 2020). Such signals often consist of endogenous intracellular molecules like cytoskeletal components, DNA, RNA, histones, and metabolites, which are passively released and inappropriately exposed to the extracellular environment. DAMPs are detected by 
immune cells that in turn activate immune pathways to mount a stress response known as sterile inflammation (Gong et al. 2020). Beyond these initial inflammatory responses, DAMPs are also important for orchestrating the subsequent steps in tissue repair and regeneration (Venereau et al. 2015). In Drosophila, the actinbinding protein $\alpha$-actinin has been shown to act as a DAMP when purified and injected into adult flies, inducing a humoral immune response in the form of cytokine signal activation specifically in the fat body (Srinivasan et al. 2016; Gordon et al. 2018). Similarly, apoptotic-deficient Drosophila constitutively activate immune signaling in the larval fat body due to the presence of circulating DAMPs in the hemolymph (Ming et al. 2014). Recently, it was shown that imaginal disc damage leads to the upregulation of circulating metabolites produced by the fat body, which are necessary for proper disc repair (Kashio and Miura 2020), highlighting the importance of interorgan communication in the context of regeneration. Thus, one possibility is that necrotic cells release DAMP signals to induce pro-regenerative NiA in the disc via an immune response involving the fat body. The role of hemocytes, an essential population of immune cells, in the regeneration of damaged imaginal discs has also been explored following physical and apoptotic damage (Katsuyama and Paro 2013; Pastor-Pareja et al. 2008; Fogarty et al. 2016), and in the context of sterile inflammation that occurs following necrosis (Shaukat et al. 2015). Considering the vital roles that hemocytes play in these processes it is possible they also mediate immune or other signals to induce NiA formation. Alternatively, DAMPs are also known to have stimulatory effects on non-immune cells (Chen and Nunez 2010; Gong et al. 2020), and thus could be sensed directly by the immediately surrounding pouch tissues to induce NiA. Going forward, it will be important to ascertain both the identity of the signal and the physiological mechanism by which necrosis leads to non-autonomous cell death in the wing disc.

\section{$\mathrm{NiA}$ is necessary for proliferation and subsequent regeneration}

Our data show that blocking NiA following necrosis inhibits the localized proliferation that promotes tissue repair, strongly suggesting that NiA is a vital part of the recovery process. How this type of cell death might promote proliferation is still unclear, however. AiP is a well-established phenomenon, in which dying cells release mitogens, such as wg and dpp, downstream of JNK signaling to stimulate localized proliferation (Perez-Garijo and Steller 2015; Fogarty and Bergmann 2017). These mitogens act via a feed-forward mechanism that involves the release of ROS, recruitment of hemocytes and induction of further JNK signaling to promote repair (Fogarty et al. 2016; Fogarty and Bergmann 2017; Amcheslavsky et al. 2018). In contrast, we have shown that cells undergoing NiA do not detectably express $w g$ or dpp, even in an undead state, and do not activate JNK signaling. Although we have not ruled out other characterized mitogens being involved, these findings suggest that NiA may not promote proliferation via the established AiP mechanism. An important consideration is whether cells undergoing NiA release specific signals to direct tissue regrowth, as seen with AiP, or if the tissue loss resulting from necrosis and NiA is instead sensed more generally at the level of the whole disc, stimulating coordinated regrowth in addition to localized proliferation (Hariharan 2015). The identification of signals produced by NiA cells and a better understanding of proliferation rates across a recovering disc will help to reveal the contribution of these different mechanisms to necrosis-induced regeneration.
Tissue necrosis is associated with many types of traumatic injury and diseases in humans, including ischemic injuries, infections, and cancer. Using model systems like the one we have developed here to understand how necrotic cells impact neighboring healthy tissues may help to identify therapeutic interventions that promote healing in tissues which otherwise struggle to recover from necrosis, such as those of humans.

\section{Data and reagent availability}

Strains and plasmids are available upon request, and details of stocks and reagents used in this study are available in the Reagent Table. The authors affirm that all data necessary for confirming the conclusions of the article are present within the article, figures, and tables. Supplemental Material available at figshare: https://doi.org/10.25386/genetics.15127761.

\section{Acknowledgments}

The authors would like to thank Dr. David Bilder and Dr. Iswar Hariharan of UC Berkeley and Dr. Tian Xie of the Stowers Institute for their gift of stocks. We thank John Weston Quinn, Ayla Zustra and current members of the Harris lab for useful input and feedback. We thank the Bloomington Stock Center, Developmental Studies Hybridoma Bank, and BestGene Inc. for stocks, reagents, and services.

\section{Funding}

This work was supported by a grant from the Eunice Kennedy Shriver National Institute of Child Health and Human Development (NICHD) 1 R21 HD102765-01 to Robin Harris.

\section{Conflicts of interest}

The authors declare that there is no conflict of interest.

\section{Literature cited}

Adachi-Yamada T, Fujimura-Kamada K, Nishida Y, Matsumoto K. 1999. Distortion of proximodistal information causes JNK-dependent apoptosis in Drosophila wing. Nature. 400: 166-169.

Amcheslavsky A, Wang S, Fogarty CE, Lindblad JL, Fan Y, et al. 2018. Plasma membrane localization of apoptotic caspases for non-apoptotic functions. Dev Cell. 45:450-464.e453.

Beira JV, Paro R. 2016. The legacy of Drosophila imaginal discs. Chromosoma. 125:573-592.

Bergmann A, Steller H. 2010. Apoptosis, stem cells, and tissue regeneration. Sci Signal. 3:re8.

Bianchi ME. 2007. DAMPs, PAMPs and alarmins: all we need to know about danger. J Leukoc Biol. 81:1-5.

Bilak A, Uyetake L, Su TT. 2014. Dying cells protect survivors from radiation-induced cell death in Drosophila. PLoS Genet. 10: e1004220.

Bonne SL, Kadri SS. 2017. Evaluation and management of necrotizing soft tissue infections. Infect Dis Clin North Am. 31:497-511.

Brock AR, Seto M, Smith-Bolton RK. 2017. Cap-n-collar promotes tissue regeneration by regulating ROS and JNK signaling in the Drosophila melanogaster wing imaginal disc. Genetics. 206: 1505-1520. 
Chatterjee N, Bohmann D. 2012. A versatile phic31 based reporter system for measuring AP-1 and Nrf2 signaling in Drosophila and in tissue culture. PLoS One. 7:e34063.

Chen GY, Nunez G. 2010. Sterile inflammation: sensing and reacting to damage. Nat Rev Immunol. 10:826-837.

Chera S, Ghila L, Dobretz K, Wenger Y, Bauer C, et al. 2009. Apoptotic cells provide an unexpected source of Wnt3 signaling to drive hydra head regeneration. Dev Cell. 17:279-289.

Everetts NJ, Worley MI, Yasutomi R, Yosef N, Hariharan IK. 2021. Single-cell transcriptomics of the Drosophila wing disc reveals instructive epithelium-to-myoblast interactions. Elife. 10:

Fogarty CE, Bergmann A. 2015. The sound of silence: signaling by apoptotic cells. Curr Top Dev Biol. 114:241-265.

Fogarty CE, Bergmann A. 2017. Killers creating new life: caspases drive apoptosis-induced proliferation in tissue repair and disease. Cell Death Differ. 24:1390-1400.

Fogarty CE, Diwanji N, Lindblad JL, Tare M, Amcheslavsky A, et al. 2016. Extracellular reactive oxygen species drive apoptosis-induced proliferation via Drosophila macrophages. Curr Biol. 26:575-584.

Fox DT, Cohen E, Smith-Bolton R. 2020. Model systems for regeneration: Drosophila. Development. 147:

Fuchs Y, Steller H. 2011. Programmed cell death in animal development and disease. Cell. 147:742-758.

Fuchs Y, Steller H. 2015. Live to die another way: modes of programmed cell death and the signals emanating from dying cells. Nat Rev Mol Cell Biol. 16:329-344.

Gong T, Liu L, Jiang W, Zhou R. 2020. DAMP-sensing receptors in sterile inflammation and inflammatory diseases. Nat Rev Immunol. 20:95-112.

Gordon O, Henry CM, Srinivasan N, Ahrens S, Franz A, et al. 2018. Alpha-actinin accounts for the bioactivity of actin preparations in inducing stat target genes in Drosophila melanogaster. Elife. 7:

Grasl-Kraupp B, Ruttkay-Nedecky B, Koudelka H, Bukowska K, Bursch W, et al. 1995. In situ detection of fragmented DNA (TUNEL assay) fails to discriminate among apoptosis, necrosis, and autolytic cell death: a cautionary note. Hepatology. 21: 1465-1468.

Hacker G. 2018. Apoptosis in infection. Microbes Infect. 20:552-559.

Hakkarainen TW, Kopari NM, Pham TN, Evans HL. 2014. Necrotizing soft tissue infections: review and current concepts in treatment, systems of care, and outcomes. Curr Probl Surg. 51:344-362.

Hariharan IK. 2015. Organ size control: lessons from Drosophila. Dev Cell. 34:255-265.

Hariharan IK, Serras F. 2017. Imaginal disc regeneration takes flight. Curr Opin Cell Biol. 48:10-16.

Harris RE, Setiawan L, Saul J, Hariharan IK. 2016. Localized epigenetic silencing of a damage-activated Wnt enhancer limits regeneration in mature Drosophila imaginal discs. Elife. 5:e11588.

Harris RE, Stinchfield MJ, Nystrom SL, McKay DJ, Hariharan IK. 2020. Damage-responsive, maturity-silenced enhancers regulate multiple genes that direct regeneration in Drosophila. Elife. 9:e58305.

Hay BA, Wolff T, Rubin GM. 1994. Expression of baculovirus p35 prevents cell death in Drosophila. Development. 120:2121-2129.

Huh JR, Guo M, Hay BA. 2004. Compensatory proliferation induced by cell death in the Drosophila wing disc requires activity of the apical cell death caspase Dronc in a nonapoptotic role. Curr Biol. 14:1262-1266

Kashio S, Miura M. 2020. Kynurenine metabolism in the fat body non-autonomously regulates imaginal disc repair in Drosophila. iScience. 23:101738

Katsuyama T, Paro R. 2013. Innate immune cells are dispensable for regenerative growth of imaginal discs. Mech Dev. 130: 112-121.
Khan SJ, Abidi SNF, Skinner A, Tian Y, Smith-Bolton RK. 2017. The Drosophila Duox maturation factor is a key component of a positive feedback loop that sustains regeneration signaling. PLoS Genet. 13:e1006937.

Kohda K, Wang Y, Yuzaki M. 2000. Mutation of a glutamate receptor motif reveals its role in gating and delta2 receptor channel properties. Nat Neurosci. 3:315-322.

Konstantinidis K, Whelan RS, Kitsis RN. 2012. Mechanisms of cell death in heart disease. Arterioscler Thromb Vasc Biol. 32: 1552-1562.

La Marca JE, Richardson HE. 2020. Two-faced: roles of JNK signalling during tumourigenesis in the Drosophila model. Front Cell Dev Biol. 8:42.

Li F, Huang Q, Chen J, Peng Y, Roop DR, et al. 2010. Apoptotic cells activate the "phoenix rising" pathway to promote wound healing and tissue regeneration. Sci Signal. 3:ra13.

Liu K, Ding L, Li Y, Yang H, Zhao C, et al. 2014. Neuronal necrosis is regulated by a conserved chromatin-modifying cascade. Proc Natl Acad Sci U S A. 111:13960-13965.

Martin FA, Perez-Garijo A, Morata G. 2009. Apoptosis in Drosophila: compensatory proliferation and undead cells. Int J Dev Biol. 53: 1341-1347.

Masi L, Falchetti A, Brandi ML. 2007. Osteonecrosis in genetic disorders. Clin Cases Miner Bone Metab. 4:27-29.

Milner PF, Kraus AP, Sebes JI, Sleeper LA, Dukes KA, et al. 1991. Sickle cell disease as a cause of osteonecrosis of the femoral head. $\mathrm{N}$ Engl J Med. 325:1476-1481.

Ming M, Obata F, Kuranaga E, Miura M. 2014. Persephone/spatzle pathogen sensors mediate the activation of toll receptor signaling in response to endogenous danger signals in apoptosis-deficient Drosophila. J Biol Chem. 289:7558-7568.

Pastor-Pareja JC, Wu M, Xu T. 2008. An innate immune response of blood cells to tumors and tissue damage in Drosophila. Dis Model Mech. 1:144-154; discussion 153.

Patel S. 2018. Danger-associated molecular patterns (DAMPs): the derivatives and triggers of inflammation. Curr Allergy Asthma Rep. 18:63.

Pellettieri J, Fitzgerald P, Watanabe S, Mancuso J, Green DR, et al. 2010. Cell death and tissue remodeling in planarian regeneration. Dev Biol. 338:76-85.

Perez-Garijo A, Fuchs Y, Steller H. 2013. Apoptotic cells can induce non-autonomous apoptosis through the TNF pathway. Elife. 2: e01004.

Perez-Garijo A, Martin FA, Morata G. 2004. Caspase inhibition during apoptosis causes abnormal signalling and developmental aberrations in Drosophila. Development. 131:5591-5598.

Perez-Garijo A, Steller H. 2015. Spreading the word: nonautonomous effects of apoptosis during development, regeneration and disease. Development. 142:3253-3262.

Pinal N, Calleja M, Morata G. 2019. Pro-apoptotic and pro-proliferation functions of the JNK pathway of Drosophila: roles in cell competition, tumorigenesis and regeneration. Open Biol. 9:180256.

Radak D, Katsiki N, Resanovic I, Jovanovic A, Sudar-Milovanovic E, et al. 2017. Apoptosis and acute brain ischemia in ischemic stroke. Curr Vasc Pharmacol. 15:115-122.

Roh JS, Sohn DH. 2018. Damage-associated molecular patterns in inflammatory diseases. Immune Netw. 18:e27.

Ryoo HD, Gorenc T, Steller H. 2004. Apoptotic cells can induce compensatory cell proliferation through the jnk and the wingless signaling pathways. Dev Cell. 7:491-501.

Santabarbara-Ruiz P, Esteban-Collado J, Perez L, Viola G, Abril JF, et al. 2019. Ask1 and Akt act synergistically to promote ros-dependent regeneration in Drosophila. PLoS Genet. 15:e1007926. 
Santabarbara-Ruiz P, Lopez-Santillan M, Martinez-Rodriguez I, Binagui-Casas A, Perez L, et al. 2015. ROS-induced JNK and p38 signaling is required for unpaired cytokine activation during Drosophila regeneration. PLoS Genet. 11:e1005595.

Shaukat Z, Liu D, Gregory S. 2015. Sterile inflammation in Drosophila. Mediators Inflamm. 2015:369286.

Srinivasan N, Gordon O, Ahrens S, Franz A, Deddouche S, et al. 2016. Actin is an evolutionarily-conserved damage-associated molecular pattern that signals tissue injury in Drosophila melanogaster. Elife. 5:

Su TT. 2015. Non-autonomous consequences of cell death and other perks of being metazoan. AIMS Genet. 2:54-69.

Su TT. 2020. Non-apoptotic roles of apoptotic proteases: new tricks for an old dog. Open Biol. 10:200130.

Suzanne M, Steller H. 2013. Shaping organisms with apoptosis. Cell Death Differ. 20:669-675.

Tseng AS, Adams DS, Qiu D, Koustubhan P, Levin M. 2007. Apoptosis is required during early stages of tail regeneration in Xenopus laevis. Dev Biol. 301:62-69.
Venereau E, Ceriotti C, Bianchi ME. 2015. DAMPs from cell death to new life. Front Immunol. 6:422.

Vriz S, Reiter S, Galliot B. 2014. Cell death: a program to regenerate. Curr Top Dev Biol. 108:121-151.

Wlodkowic D, Telford W, Skommer J, Darzynkiewicz Z. 2011. Apoptosis and beyond: cytometry in studies of programmed cell death. Methods Cell Biol. 103:55-98.

Wong RS. 2011. Apoptosis in cancer: from pathogenesis to treatment. J Exp Clin Cancer Res. 30:87.

Worley MI, Setiawan L, Hariharan IK. 2012. Regeneration and transdetermination in Drosophila imaginal discs. Annu Rev Genet. 46: 289-310.

Yang Y, Hou L, Li Y, Ni J, Liu L. 2013. Neuronal necrosis and spreading death in a Drosophila genetic model. Cell Death Dis. 4: e723.

Yuan J. 2009. Neuroprotective strategies targeting apoptotic and necrotic cell death for stroke. Apoptosis. 14:469-477.

Communicating editor: H. Jafar-Nejad 